Published in final edited form as:

Am J Prev Med. 2019 January ; 56(1): 116-124. doi:10.1016/j.amepre.2018.07.037.

\title{
Optimism and Healthy Aging in Women
}

\author{
Peter James, ScD ${ }^{1}$, Eric S. Kim, $\mathrm{PhD}^{2,3}$, Laura D. Kubzansky, $\mathrm{PhD}^{2,3}$, Emily S. Zevon, \\ $\mathrm{ScD}^{2,3}$, Claudia Trudel-Fitzgerald, $\mathbf{P h D}^{2,3}$, and Francine Grodstein, $\mathbf{S c D}^{4,5}$ \\ ${ }^{1}$ Department of Population Medicine, Harvard Medical School and Harvard Pilgrim Health Care \\ Institute, Boston, Massachusetts; \\ ${ }^{2}$ Department of Social and Behavioral Sciences, Harvard T.H. Chan School of Public Health, \\ Boston, Massachusetts; \\ ${ }^{3}$ Lee Kum Sheung Center for Health and Happiness, Harvard T.H. Chan School of Public Health, \\ Boston, Massachusetts; \\ ${ }^{4}$ Department of Epidemiology, Harvard T.H. Chan School of Public Health, Boston, \\ Massachusetts; \\ ${ }^{5}$ Channing Division of Network Medicine, Brigham and Women's Hospital, Boston, \\ Massachusetts
}

\section{Abstract}

Introduction: Optimism - the expectation that good things will happen-has emerged as a promising health asset, as it appears to be related to healthier behaviors and reduced disease risk. Growing research finds that higher optimism is associated with lower mortality, yet it is critical to understand whether this prolonged longevity is accompanied by good health. This study tested whether higher optimism was associated with increased likelihood of healthy aging.

Methods: Prospective data analyzed in 2018 from the Nurses' Health Study included 33,326 women with no major chronic diseases at baseline. Poisson regression models evaluated if optimism was associated with healthy aging 8 years later, considering potential confounders (sociodemographic variables, depression) and intermediate variables (health behaviors). Optimism was assessed in 2004 by validated self-report using mailed questionnaires and healthy aging was assessed in 2012, defined as: (1) remaining free of major chronic diseases, (2) having no subjective memory impairment, (3) having intact physical function, and (4) surviving through follow-up.

Results: Overall, $20.5 \%$ of women $(n=6,823)$ fulfilled the definition of healthy aging in 2012. After adjusting for sociodemographic factors and depression, the most (top quartile) versus least (bottom quartile) optimistic women had a $23 \%$ greater likelihood of healthy aging (95\% CI=1.16,

Address correspondence to: Peter James, ScD, Division of Chronic Disease Research Across the Lifecourse (CoRAL), Department of Population Medicine, Harvard Medical School and Harvard Pilgrim Health Care Institute, Landmark Center 401 Park Drive, Suite 401, Boston MA 02215.pjames@ hsph.harvard.edu.

Publisher's Disclaimer: This is a PDF file of an unedited manuscript that has been accepted for publication. As a service to our customers we are providing this early version of the manuscript. The manuscript will undergo copyediting, typesetting, and review of the resulting proof before it is published in its final citable form. Please note that during the production process errors may be discovered which could affect the content, and all legal disclaimers that apply to the journal pertain. 
1.30). Associations were similar in white and black participants, although the sample of black women was small $(n=354)$.

Conclusions: Higher optimism was associated with increased likelihood of healthy aging, suggesting that optimism, a potentially modifiable health asset, merits further research for its potential to improve health in aging.

\section{INTRODUCTION}

There are 46.3 million people aged more than 65 years in the U.S., a figure projected to increase nearly $50 \%$ in 15 years. ${ }^{1}$ Chronic disease burden rises with age, and although average life expectancies have increased, years lost to disability has also increased. ${ }^{2}$ Thus, identifying factors that contribute to healthy aging is vital for improving health and wellbeing in older people and containing healthcare costs. ${ }^{3}$ Recent research suggests there may be modifiable health resources, or assets, that reduce risks of chronic diseases of aging. Dispositional optimism — the generalized expectation that good things will happen—has emerged as a promising health asset. Optimism contributes to an individual's capacity to transform goals into behaviors, and studies suggest that these health effects are independent of psychological distress (e.g., depression). Importantly, although optimism is shaped by social structural factors (e.g., SES) and is approximately $25 \%$ heritable, ${ }^{4,5}$ randomized trials suggest optimism can be modified using approaches including cognitive-behavioral therapy, as well as less intensive classroom-style activities and brief paper and pencil exercisessome of which are inexpensive and accessible. ${ }^{6-9}$ Optimism has been associated with healthier behaviors (e.g., diet, medication adherence), ${ }^{10-13}$ improved biologic functioning (e.g., less inflammation, healthier antioxidant levels), ${ }^{14-16}$ and reduced risk of chronic diseases ${ }^{17,18}$ and mortality. ${ }^{19,20}$ Yet, it remains largely unknown whether any prolonged longevity is accompanied by overarching health.

To assess the relation of optimism to healthy aging, data from a large cohort of women were used to evaluate if higher optimism is associated with increased likelihood of healthy aging among those who survive to older ages; analyses controlled for past/current depression and other relevant covariates. Finally, because some work suggests that health effects of optimism are similar by race, ${ }^{18,19}$ analyses evaluated if associations are similar in black and white women.

\section{METHODS}

\section{Study Population}

The Nurses' Health Study began in 1976 when registered female nurses, ages 30-55 years, completed a mailed questionnaire on their health and lifestyle. Since then, questionnaires have been mailed to participants biennially. Follow-up remains $\cong 90 \%$. A measure of optimism was included in the 2004 questionnaire; thus, 2004 was considered baseline for this study. Healthy aging was assessed in 2012, when measurements were first available for all relevant components including chronic disease diagnoses, cognitive function, and physical function. Women who died before study baseline in $2004(n=30,848)$, or reported a history of major chronic diseases (described below) in 2004 or on prior questionnaires 
$(n=34,693)$ were excluded. Women were also excluded if they were persistent nonresponders, as they were sent a shorter version of the questionnaire that did not assess optimism or other key variables $(n=8,802)$. Women were further excluded if they did not answer optimism questions in $2004(n=2,477)$ and either did not respond to questions on healthy aging in 2012 or died before $2012(n=11,555)$. This yielded an analytic sample of $n=33,326$. When comparing women with versus without relevant data, major characteristics were similar (e.g., excluded participants were slightly older (aged 71 vs 68 years), had nearly identical BMI (25.8 vs $\left.26.0 \mathrm{~kg} / \mathrm{m}^{2}\right)$, and had similar prevalence of smoking (11\% vs $9 \%)$.

The study was approved by the IRB at the Brigham and Women's Hospital.

\section{Measures}

Optimism was assessed in 2004 using the Life Orientation Test-Revised. The measure has good psychometric properties. ${ }^{21}$ Using a 5-point Likert scale, participants were asked the degree to which they agreed with six statements. After reverse coding negatively worded items, all items were summed to create a composite score ranging from zero to 24 , with higher scores indicating greater optimism; to facilitate comparisons of effect size across studies, scores were standardized (mean $=0, \mathrm{SD}=1$ ). Because optimism is best characterized by endorsing positively worded items and rejecting negatively worded items, recent recommendations to use the 6 -item composite were used rather than 3 -item subscales. ${ }^{22}$ To assess discontinuous or threshold effects, quartiles of optimism were created based on this sample's distribution.

To obtain a multisystem view of healthy aging, healthy versus usual aging was defined based on the model of successful aging by Rowe and Kahn, ${ }^{23}$ a comprehensive perspective that includes comorbidities and disabilities. These domains were assessed on the 2012 follow-up questionnaire. ${ }^{24,25}$ Women were categorized as healthy agers if they survived to the 2012 questionnaire (i.e., aged $>65$ years), were free of major chronic diseases, had no subjective memory impairment, and had no major physical limitations. Women who survived through 2012 but did not meet these criteria were considered usual agers. Healthy aging was categorized as a binary variable (yes/no). The authors also considered healthy versus usual aging across a broader continuum, characterized by the number of domains in which an individual was healthy (range, $0-3$ ).

Recognizing that varying cutpoints can be used in each domain to determine healthy versus usual aging, several alternate cutpoints were previously tested; associations between known risk factors and healthy aging were maintained across variations in cutpoints, indicating that results are robust to various healthy aging definitions. ${ }^{26}$ Though healthy aging definitions can include mental health, analyses did not consider this domain because it is linked to optimism itself. Below is the definition of "healthy" for each domain.

Eleven chronic diseases were considered that are primary causes of mortality in the U.S. or are highly debilitating; disease diagnosis was reported on biennial questionnaires through 2012. Diseases included cancer (other than non-melanoma skin cancer), myocardial infarction, coronary artery bypass surgery or percutaneous transluminal coronary 
angioplasty, congestive heart failure, stroke, type 2 diabetes, kidney failure, chronic obstructive pulmonary disease, Parkinson disease, multiple sclerosis, and amyotrophic lateral sclerosis. Women who reported no history of these diseases as of 2012 were considered "healthy." Previous work showed high validity of self-reported chronic disease diagnoses in cohort participants. ${ }^{27}$

Participants were asked seven (yes/no) questions to assess changes in memory and related tasks: general memory, remembering short lists (e.g., shopping), remembering things from one second to the next, recent events, understanding or following verbal instructions, following a group conversation or plot in a TV program (because of memory loss), and navigating familiar streets. These items were based on the Structured Telephone Interview for Dementia Assessment ${ }^{28}$; these subjective memory items showed strong relations with objective cognitive tests in an Nurses' Health Study subsample. ${ }^{29}$ Further, research in other cohorts has established that subjective memory concerns correlate well with clinical diagnosis of dementia and dementia pathology. ${ }^{30}$ Research on healthy aging often includes subjective memory concerns because of their relevance for quality of life. Participants reporting one or less of the seven reported memory concerns were considered "healthy."

Physical function was assessed on the 2012 questionnaire using the Medical Outcomes Short Form-36 physical function items. Physical function limitations were defined based on ten items encompassing activities of daily living ranging from moderate (e.g., vacuuming, bathing) to difficult (e.g., running, lifting heavy objects). Participants reporting no physical function limitations were considered "healthy." Sociodemographic variables were obtained from the 2004 questionnaire or from the previous questionnaire if not available in 2004, and included: age (continuous), race (white, black, Asian, other), marital status (married, divorced/separated/single, widowed), nurse's education (registered nurse/associate's degree, bachelor's, master's/doctorate; assessed in 1992), husband's education (<high school, some high school, high school graduate, college graduate, graduate school; assessed in 1992). Neighborhood-level SES was assessed through census tract median income. Past/current depression (yes/no) was defined according to self-report of physician diagnosis or regular use of antidepressants on any questionnaire from 2004 or earlier, or a score of ten or more on the validated Center for Epidemiologic Study Depression Scale Revised in 2004. ${ }^{31}$ Baseline physical function was assessed in 2004.

Health-related behaviors can be considered potential confounding variables or intermediate factors. These included BMI, diet, physical activity, cigarette smoking, alcohol intake, and annual physical exams, all queried in 2002-2004. BMI was calculated from self-reported weight and height. Diet was assessed with the Willett Food Frequency Questionnaire; diet quality was quantified using the Alternative Healthy Eating Index-2010. Modified to exclude alcohol, because analyses controlled for alcohol, the Alternative Healthy Eating Index-2010 ranged from zero to 100 and included ten diet components. ${ }^{32}$ Physical activity was assessed across six types of exercise, previously validated in this cohort and summarized as weekly expenditure of METs ( $<3,3$ to $<9,9$ to $<18,18$ to $<27, \geq 27$ METs/ week). Reported cigarette smoking was characterized as never, former, current smoker. Reported alcohol intake from wine, beer, and liquor was combined (none, more than zero to 
$14, \geq 15$ grams/day). Participants reported annual physical exams for screening purposes (yes/no).

\section{Statistical Analysis}

Because the probability of healthy aging was not rare (i.e., >10\%), Poisson regression models with robust variance were used. Primary analyses used quartiles of optimism; a continuous variable with median score for each optimism quartile was used to estimate $p$ values for trends. Optimism was also considered as a continuous variable indicating the change in likelihood of healthy aging as a function ofa one SD increase in optimism. Three sets of models were evaluated. The first was age adjusted. The second adjusted for potential confounding factors, adding race, marital status, nurse's education, husband's education, neighborhood SES, current/past depression, and baseline physical function levels. A third model added potential intermediates, including BMI, diet, physical activity, cigarette smoking, alcohol intake, and annual physical exams.

Several secondary analyses were conducted. The authors evaluated whether the association of optimism and healthy aging persisted when considering a larger spectrum of health rather than a single cutpoint for "healthy" versus "usual" aging. This was done by creating an aging score that ranged from zero to three, which captured the number of domains in which a participant qualified as healthy. Thus, a score of three represents healthy agers (i.e., healthy in all three domains), and zero to two represents a spectrum across usual aging (e.g., zero=usual agers unhealthy in all three domains). Using this score, the mean optimism levels were examined at baseline for each level of the aging score. Multinomial logistic regression was also conducted to test if optimism was associated with the aging score levels, comparing healthy agers (score=three) to each of the other aging scores (zero, one, two). To test residual confounding because of depression or physical function status at baseline, women with previous/current depression at baseline or poor physical function at baseline were also excluded. (The latter women were included in primary analyses because some had improved physical function over time and qualified as healthy agers.) Further analyses were conducted in white $(n=32,632)$ and black $(n=354)$ women. To test effect modification by race, data were pooled and an interaction term for race X continuous optimism was tested. Finally, penalized spline models evaluated potential deviations from linearity in the optimism and healthy aging association. All analyses were conducted in 2018 using SAS, version 9.3.

\section{RESULTS}

The average age at baseline was 68 years and $75 \%$ of participants were married (Table 1). At baseline, sociodemographic and health characteristics were similarly distributed across optimism quartiles (Table 1). However, women in the highest versus lowest optimism quartile had more education (14\% vs $7 \%$ with a graduate degree), higher levels of physical activity (mean 27 vs 21 MET hours/week), and substantially lower prevalence of depression (13\% vs $42 \%)$.

Strong associations existed between greater optimism and higher likelihood of healthy aging (Table $2 ; p_{\text {trend }}<0.001$ ). For example, after controlling for confounders, there was a $23 \%$ greater likelihood of healthy aging for women in the top versus bottom optimism quartile 
$(\mathrm{RR}=1.23,95 \% \mathrm{CI}=1.16,1.30)$. Adding health behaviors slightly attenuated the primary association ( $\mathrm{RR}=1.21,95 \% \mathrm{CI}=1.14,1.28)$. Considering optimism as a continuous variable, each SD increase was associated with a RR of $1.07(95 \% \mathrm{CI}=1.05,1.10)$ for healthy aging, in the fully adjusted model (Table 2).

Average optimism scores were similar in blacks and whites (mean scores were 20.3 and 19.3, respectively). In addition, black women compared with white women had more education ( $25 \%$ vs $11 \%$ with a graduate degree), lower neighborhood-level SES (16\% vs $25 \%$ in the highest quartile), and were less likely to have depression (16\% vs $24 \%$; Appendix Table 1). After adjusting for confounders (Table 3), there was a $42 \%$ greater likelihood of healthy aging with each SD increase in optimism in black women $(95 \%$ $\mathrm{CI}=1.01,1.98$ ); adding potential intermediates slightly attenuated this finding. Findings were similar in white women, albeit of a lower magnitude ( $\mathrm{RR}=1.08,95 \% \mathrm{CI}=1.06,1.10)$. An interaction test between race $\mathrm{X}$ optimism was not significant $\left(p_{\text {interaction }}=0.10\right)$.

In secondary analyses, the authors observed evidence of a graded relationship between increasingly higher optimism scores and better aging scores; individuals with an aging score of either zero, one, two (usual aging), or three (healthy aging), based on number of domains in which they were categorized as healthy, displayed the following mean optimism scores, respectively: 18.0 (zero), 18.7 (one), 19.4 (two), and 20.2 (three). Further, in age-adjusted multinomial logistic regression models, each one SD increase in optimism was significantly associated with a $15 \%$ greater odds of being a healthy ager in all three domains compared with being an ager with two healthy domains; the odds were even greater when healthy aging (score of three, healthy in all domains) was compared with being healthy in one or zero domains (i.e., $34 \%$ and $62 \%$, respectively; Appendix Table 2). The association between each SD increase in optimism and healthy aging remained when restricting to women without prior/current depression at baseline ( $\mathrm{RR}=1.08,95 \% \mathrm{CI}=1.06,1.11$, adjusted for confounders; Appendix Table 3) and those without physical function limitations at baseline $(\mathrm{RR}=1.07,95 \% \mathrm{CI}=1.04,1.09$, adjusted for confounders; Appendix Table 4). In the penalized spline model (Figure 1), the association between optimism and healthy aging remained similar across optimism scores of zero to 20; above this score (21-24), associations became somewhat stronger.

\section{DISCUSSION}

Higher optimism levels were associated with greater likelihood of healthy aging in women who survived to older ages, suggesting optimism is related not only with prolonged longevity ${ }^{19,20}$ but also with better overall health and function in those who survive. Moreover, findings appeared similar in white and black women, suggesting that optimism may serve as a health asset across individuals of different race/ethnicities. These associations controlled for depression and other potential confounders and were maintained after considering behavioral variables. It is noteworthy that these findings did not suggest associations between optimism and healthy aging are primarily driven by extreme categories of healthy versus usual aging but rather persist across the spectrum of health in aging. Results from spline models also suggested that the association of optimism with healthy aging was monotonic throughout the spectrum of optimism scores, yet became somewhat 
stronger in the upper range, indicating modest changes in optimism could still be meaningful.

These findings are compatible with previous research showing associations between higher optimism and reduced risk of age-related conditions (e.g., heart disease, cognitive impairment), and mortality from age-related diseases. ${ }^{19,20}$ Findings are also supported by studies examining optimism in relation to health-related behaviors contributing to healthy aging, in diverse populations. For example, a cross-sectional study of older adults reported inverse associations of optimism with smoking, physical activity, diet, and weight status in blacks and whites, ${ }^{18}$ and a prospective study found optimism was associated with better diet quality and improvement in diet over time in white and black women. ${ }^{10}$ The current study specifically addresses a multidimensional set of components vital for aging well and thriving, and suggests optimism is a broadly applicable health asset across black and white women. However, these findings require replication in other diverse samples.

Mechanisms underlying the health-promoting effects of optimism could include both indirect (e.g., health behaviors) and direct effects (e.g., altered biological function). For example, people with higher optimism may be proactive in managing their health and engage in healthier behaviors. ${ }^{10-13}$ In the present study, associations were modestly attenuated after accounting for health behaviors, suggesting other mechanisms are likely at play. Research shows those with higher optimism display healthier regulation of physiological systems (e.g., healthier lipid profiles, antioxidants, immune responsiveness $)^{14-16}$ associated with healthy aging; these direct mechanisms merit research.

\section{Limitations}

The current study has limitations. The use of self-report to measure healthy aging could contribute measurement error; however, studies have documented validity and accuracy in reporting these conditions, especially in populations of health professionals. ${ }^{33}$ Additionally, the definition of healthy aging was achieved by a minority of the study population. Although there is no standard definition of healthy aging, previous research has tested various cutpoints to define healthy aging across the three domains, and associations of risk factors to healthy aging remained robust. ${ }^{26}$ Confounding by unmeasured variables is possible. However, a range of confounding variables were considered, and the somewhat homogeneous population may additionally limit key confounding factors (e.g., healthcare knowledge and access). Because only women were included, generalizability of these findings may be limited. Nonetheless, because women live longer than men, understanding factors related to healthy aging in women has particular public health relevance. Reverse causation is possible if underlying physical, mental, or cognitive health conditions influenced optimism at baseline. However, such concerns may be minimized given the long lag between measurement of optimism and healthy aging, exclusion of those with chronic disease at baseline, adjustment for factors including baseline physical function, and analyses excluding anyone with mental health or physical function limitations at baseline. Further, psychological well-being appears stable in the face of declining health and news of impending health decline. For example, several studies have indicated that optimism is largely unchanged before and after cancer diagnosis and treatment. ${ }^{34,35}$ 


\section{CONCLUSIONS}

Optimism appears strongly associated with greater likelihood of healthy aging. As the population ages, a range of interventions will be necessary to improve health. These overall findings, including those revealing similar relations in white and black women, suggest that optimism may be a promising target for intervention among diverse populations. Early randomized trials suggest that optimism can be altered with a variety of interventions. ${ }^{6}$ Lowintensity interventions that do not require provider training (e.g., counting blessings, acts of kindness) may increase optimism, although effects appear modest. ${ }^{6}$ Other more intensive interventions show promise. ${ }^{8,9}$ For example, a study among heart disease patients used a classroom-style approach with eight sessions of multiple exercises and reported a $25 \%$ increase in optimism right after the intervention period and a $15 \%$ increase in optimism levels at the 8-week follow-up assessment, compared with the control group. ${ }^{9}$ The present study suggests the importance of further developing approaches that foster optimism among older adults, with the goal of enhancing trajectories of health and well-being.

\section{Supplementary Material}

Refer to Web version on PubMed Central for supplementary material.

\section{ACKNOWLEDGMENTS}

Both P.J. and E.S.K. contributed equally as first authors.

This work was supported by grants from NIH (R01AG053273, P01 CA87969, UM1CA186107, R00CA201542, T32HL098048, 3R25CA057711) and by a postdoctoral fellowship from the Fonds de Recherche du Québec - Santé to Claudia Trudel-Fitzgerald.

We would like to thank the participants and staff of the Nurses' Health Study for their valuable contributions as well as the following state cancer registries for their help: Alabama, Arizona, Arkansas, California, Colorado, Connecticut, Delaware, Florida, Georgia, Idaho, Illinois, Indiana, Iowa, Kentucky, Louisiana, Maine, Maryland, Massachusetts, Minnesota, Nebraska, New Hampshire, New Jersey, New York, North Carolina, North Dakota, Ohio, Oklahoma, Oregon, Pennsylvania, Rhode Island, South Carolina, Tennessee, Texas, Virginia, Washington, and Wyoming. The authors assume full responsibility for analyses and interpretation of these data.

Drs. James, Kubzansky, Kim, Grodstein, Trudel-Fitzgerald, and Zevon had full access to all the data in the study and take responsibility for the integrity of the data and the accuracy of the data analysis. Drs. James and Kim contributed to drafting the manuscript. All authors contributed to the study concept and design; acquisition, analysis, or interpretation of data; and critical revision of the manuscript for important intellectual content.

No financial disclosures were reported by the authors of this paper.

\section{REFERENCES}

1. Colby SL, Ortman JM. Projections of the Size and Composition of the U.S. Population. U.S. Census Bureau; 2014 www.census.gov/library/publications/2015/demo/p25-1143.html. Accessed March 20, 2017.

2. Salomon JA, Wang H, Freeman MK, et al. Healthy life expectancy for 187 countries, 1990-2010: a systematic analysis for the Global Burden Disease Study 2010. Lancet. 2012;380(9859):2144-2162. 10.1016/S0140-6736(12)61690-0. [PubMed: 23245606]

3. Oppers ES, Chikada K, Eich F, et al. The Financial Impact of Longevity Risk. International Monetary Fund; 2012. 
4. Plomin R, Scheier MF, Bergeman CS, Pedersen NL, Nesselroade JR, McClearn GE. Optimism, pessimism and mental health: a twin/adoption analysis. Pers Individ Dif. 1992;13(8):921-930. 10.1016/0191-8869(92)90009-E.

5. Boehm JK, Chen Y, Williams DR, Ryff C, Kubzansky LD. Unequally distributed psychological assets: are there social disparities in optimism, life satisfaction, and positive affect? PLoS ONE. 2015;10(2):e0118066 10.1371/journal.pone.0118066. [PubMed: 25671665]

6. Malouff JM, Schutte NS. Can psychological interventions increase optimism? A meta-analysis. J Posit Psychol. 2017;12(6):594-604. 10.1080/17439760.2016.1221122.

7. Boehm JK, Kim ES, Kubzansky LD. Positive Psychological Wellbeing In: The Routledge International Handbook of Psychosocial Epidemiology. 1st ed Routledge; 2017:156-170.

8. Celano CM, Albanese AM, Millstein RA, et al. Optimizing a positive psychology intervention to promote health behaviors following an acute coronary syndrome: the Positive Emotions after Acute Coronary Events-III (PEACE-III) randomized factorial trial. Psychosom Med. 2018;80(6):526-534. 10.1097/PSY.0000000000000584. [PubMed: 29624523]

9. Mohammadi N, Aghayousefi A, Nikrahan GR, et al. A randomized trial of an optimism training intervention in patients with heart disease. Gen Hosp Psychiatry. 2018;51:46-53. 10.1016/ j.genhosppsych.2017.12.004. [PubMed: 29316450]

10. Hingle MD, Wertheim BC, Tindle HA, et al. Optimism and diet quality in the Women's Health Initiative. J Acad Nutr Diet. 2014;114(7):1036-1045. 10.1016/j.jand.2013.12.018. [PubMed: 24556429]

11. Lemola S, Räikkönen K, Gomez V, Allemand M. Optimism and self-esteem are related to sleep. Results from a large community-based sample. Int J Behav Med. 2013;20(4):567-571. 10.1007/ s12529-012-9272-z. [PubMed: 23055029]

12. Steptoe A, Wright C, Kunz-Ebrecht SR, Iliffe S. Dispositional optimism and health behaviour in community-dwelling older people: associations with healthy ageing. Br J Health Psychol. 2006;11(1):71-84. 10.1348/135910705X42850. [PubMed: 16480556]

13. Huffman JC, Beale EE, Celano CM, et al. Effects of optimism and gratitude on physical activity, biomarkers, and readmissions after an acute coronary syndrome the Gratitude Research in Acute Coronary Events Study. Circ Cardiovasc Qual Outcomes. 2016;9(1):55-63. 10.1161/ CIRCOUTCOMES.115.002184. [PubMed: 26646818]

14. Boehm JK, Williams DR, Rimm EB, Ryff C, Kubzansky LD. Relation between optimism and lipids in midlife. Am J Cardiol. 2013;111(10):1425-1431. 10.1016/j.amjcard.2013.01.292. [PubMed: 23433765]

15. Segerstrom SC, Sephton SE. Optimistic expectancies and cell-mediated immunity: the role of positive affect. Psychol Sci. 2010;21(3):448-455. 10.1177/0956797610362061. [PubMed: 20424083]

16. Boehm JK, Williams DR, Rimm EB, Ryff C, Kubzansky LD. Association between optimism and serum antioxidants in the Midlife in the United States Study. Psychosom Med. 2013;75(1):2-10. 10.1097/PSY.0b013e31827c08a9. [PubMed: 23257932]

17. Gawronski KAB, Kim ES, Langa KM, Kubzansky LD. Dispositional optimism and incidence of cognitive impairment in older adults. Psychosom Med. 2016;78(7):819-828. 10.1097/PSY. 0000000000000345. [PubMed: 27284699]

18. Hernandez R, Kershaw KN, Siddique J, et al. Optimism and cardiovascular health: Multi-Ethnic Study of Atherosclerosis (MESA). Health Behav Policy Rev. 2015;2(1):62-73. 10.14485/HBPR. 2.1.6. [PubMed: 26213688]

19. Tindle HA, Chang Y-F, Kuller LH, et al. Optimism, cynical hostility, and incident coronary heart disease and mortality in the Women's Health Initiative. Circulation. 2009;120(8):656-662. 10.1161/CIRCULATIONAHA.108.827642. [PubMed: 19667234]

20. Kim ES, Hagan KA, Grodstein F, DeMeo DL, De Vivo I, Kubzansky LD. Optimism and causespecific mortality: a prospective cohort study. Am J Epidemiol. 2017;185(1):21-29. 10.1093/aje/ kww182. [PubMed: 27927621]

21. Scheier MF, Carver CS, Bridges MW. Distinguishing optimism from neuroticism (and trait anxiety, self-mastery, and self-esteem): a reevaluation of the Life Orientation Test. J Pers Soc Psychol. 1994;67(6):1063-1078. 10.1037/0022-3514.67.6.1063. [PubMed: 7815302] 
22. Segerstrom SC, Evans DR, Eisenlohr-Moul TA. Optimism and pessimism dimensions in the Life Orientation Test-Revised: method and meaning. J Res Personal. 2011;45(1):126-129. 10.1016/ j.jrp.2010.11.007.

23. Rowe JW, Kahn RL. Successful aging. Gerontologist. 1997;37(4):433-440. 10.1093/geront/ 37.4.433. [PubMed: 9279031]

24. Samieri C, Sun Q, Townsend MK, Rimm EB, Grodstein F. Dietary flavonoid intake at midlife and healthy aging in women. Am J Clin Nutr. 2014;100(6):1489-1497. 10.3945/ajcn.114.085605. [PubMed: 25411284]

25. Sun Q, Townsend MK, Okereke OI, Franco OH, Hu FB, Grodstein F. Physical activity at midlife in relation to successful survival in women at age 70 years or older. Arch Intern Med. 2010;170(2): 194-201. 10.1001/archinternmed.2009.503. [PubMed: 20101015]

26. Samieri C, Sun Q, Townsend MK, et al. The relation of midlife diet to healthy aging: a cohort study. Ann Intern Med. 2013;159(9):584-591. 10.7326/0003-4819-159-9-201311050-00004. [PubMed: 24189593]

27. Bao Y, Bertoia ML, Lenart EB, et al. Origin, methods, and evolution of the three Nurses' Health Studies. Am J Public Health. 2016;106(9):1573-1581. 10.2105/AJPH.2016.303338. [PubMed: 27459450]

28. Go RC, Duke LW, Harrell LE, et al. Development and validation of a Structured Telephone Interview for Dementia Assessment (STIDA): the NIMH Genetics Initiative. J Geriatr Psychiatry Neurol. 1997;10(4):161-167. 10.1177/089198879701000407. [PubMed: 9453683]

29. Amariglio RE, Townsend MK, Grodstein F, Sperling RA, Rentz DM. Specific subjective memory complaints in older persons may indicate poor cognitive function. J Am Geriatr Soc. 2011;59(9): 1612-1617. 10.1111/j.1532-5415.2011.03543.x. [PubMed: 21919893]

30. Mitchell AJ, Beaumont H, Ferguson D, Yadegarfar M, Stubbs B. Risk of dementia and mild cognitive impairment in older people with subjective memory complaints: meta-analysis. Acta Psychiatr Scand. 2014;130(6):439-451. 10.1111/acps.12336. [PubMed: 25219393]

31. Andresen EM, Malmgren JA, Carter WB, Patrick DL. Screening for depression in well older adults: evaluation of a short form of the CES-D (Center for Epidemiologic Studies Depression Scale). Am J Prev Med. 1994;10(2):77-84. 10.1016/S0749-3797(18)30622-6. [PubMed: 8037935]

32. Chiuve SE, Fung TT, Rimm EB, et al. Alternative dietary indices both strongly predict risk of chronic disease. J Nutr. 2012;142(6):1009-1018. 10.3945/jn.111.157222. [PubMed: 22513989]

33. Colditz GA, Martin P, Stampfer MJ, et al. Validation of questionnaire information on risk factors and disease outcomes in a prospective cohort study of women. Am J Epidemiol. 1986;123(5):894900. 10.1093/oxfordjournals.aje.a114319. [PubMed: 3962971]

34. Schou I, Ekeberg O, Sandvik L, Ruland CM. Stability in optimism-pessimism in relation to bad news: a study of women with breast cancer. J Pers Assess. 2005;84(2):148-154. 10.1207/ s15327752jpa8402_04. [PubMed: 15799889]

35. Stiegelis HE, Hagedoorn M, Sanderman R, van der Zee KI, Buunk BP, van den Bergh ACM. Cognitive adaptation: a comparison of cancer patients and healthy references. Br J Health Psychol. 2003;8(pt 3):303-318. 10.1348/135910703322370879. [PubMed: 14606975] 


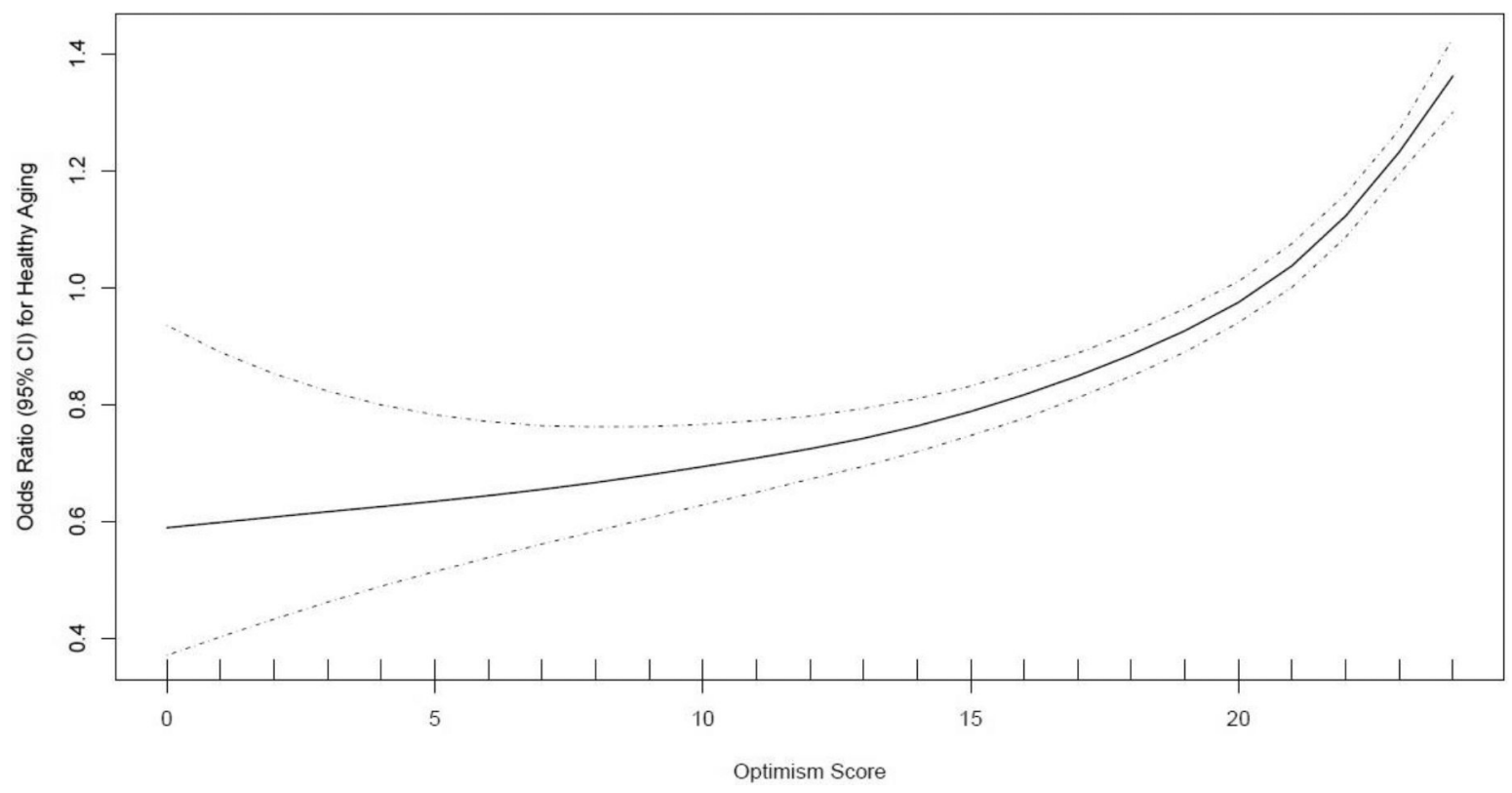

Figure 1.

Penalized spline of nonlinear relationship between continuous optimism score and odds of healthy aging. 
Table 1.

Characteristics of Participants at Baseline, Overall and by Quartiles of Optimism Score ${ }^{a, b, c}$

\begin{tabular}{|c|c|c|c|c|c|}
\hline \multirow{2}{*}{ Characteristic } & \multicolumn{5}{|c|}{ Optimism } \\
\hline & Overall (N=33,326) & Quartile $1^{d}(\mathrm{n}=8,206)$ & Quartile $2^{e}(\mathrm{n}=9,242)$ & Quartile $3_{(n=6,103)}$ & Quartile $4^{g}(\mathrm{n}=9,775)$ \\
\hline \multicolumn{6}{|l|}{ Demographic factors } \\
\hline Mean age in years (SD) & $67.9(6.4)$ & $68.3(6.6)$ & $68.3(6.4)$ & $67.8(6.3)$ & $67.4(6.2)$ \\
\hline \multicolumn{6}{|l|}{ Race, $\%$} \\
\hline White & 97.9 & 98.2 & 97.5 & 98.0 & 98.0 \\
\hline Black & 1.1 & 0.7 & 1.1 & 1.1 & 1.3 \\
\hline Asian & 0.8 & 1.0 & 1.0 & 0.7 & 0.4 \\
\hline Other & 0.3 & 0.2 & 0.3 & 0.3 & 0.3 \\
\hline \multicolumn{6}{|l|}{ Marital status, $\%$} \\
\hline Married & 75.0 & 71.8 & 74.6 & 75.8 & 77.5 \\
\hline $\begin{array}{l}\text { Divorced, separate, } \\
\text { or single }\end{array}$ & 7.7 & 8.4 & 7.7 & 7.3 & 7.2 \\
\hline Widow & 17.3 & 19.8 & 17.7 & 16.9 & 15.2 \\
\hline \multicolumn{6}{|l|}{ Education, $\%$} \\
\hline Registered nurse & 61.0 & 66.7 & 62.3 & 59.5 & 55.8 \\
\hline Bachelor's & 20.7 & 17.5 & 20.2 & 21.7 & 23.3 \\
\hline Master's or Doctorate & 10.7 & 7.1 & 9.6 & 12.0 & 13.7 \\
\hline \multicolumn{6}{|c|}{ Husbands' highest education, $\%$} \\
\hline $\begin{array}{l}\text { High school graduate } \\
\text { or less }\end{array}$ & 32.2 & 34.8 & 32.6 & 31.5 & 30.0 \\
\hline College graduate & 24.1 & 23.1 & 24.1 & 24.6 & 24.7 \\
\hline Graduate school & 22.0 & 18.6 & 21.7 & 23.2 & 24.5 \\
\hline \multicolumn{6}{|c|}{ Census tract median income quartiles, $\%$} \\
\hline$\$ 41,125$ & 25.0 & 26.7 & 24.8 & 23.8 & 24.5 \\
\hline$\$ 53,441$ & 25.0 & 25.0 & 25.3 & 24.7 & 24.9 \\
\hline$\$ 66,935$ & 25.0 & 25.4 & 25.1 & 25.5 & 24.4 \\
\hline$\$ 91,272$ & 25.0 & 22.9 & 24.8 & 26.0 & 26.2 \\
\hline \multicolumn{6}{|l|}{ Health behaviors } \\
\hline \multicolumn{6}{|l|}{ Smoking status, $\%$} \\
\hline Never smoker & 48.1 & 46.5 & 47.6 & 47.9 & 50.2 \\
\hline Past smoker & 42.5 & 42.3 & 42.8 & 43.5 & 41.8 \\
\hline Current & 8.9 & 10.7 & 9.2 & 8.1 & 7.7 \\
\hline \multicolumn{6}{|l|}{ Alcohol consumption, $\%$} \\
\hline None & 36.9 & 40.4 & 36.4 & 34.6 & 36.0 \\
\hline $1-14 \mathrm{~g} / \mathrm{d}$ & 46.9 & 44.8 & 47.3 & 48.8 & 47.2 \\
\hline$\geq 15 \mathrm{~g} / \mathrm{d}$ & 14.6 & 13.2 & 14.6 & 15.4 & 15.4 \\
\hline $\begin{array}{l}\text { Physical exam for } \\
\text { screening purposes, } \%\end{array}$ & 88.5 & 86.4 & 88.5 & 89.7 & 89.4 \\
\hline $\begin{array}{l}\text { Mean total physical } \\
\text { activity, MET/week (SD) }\end{array}$ & $23.9(26.9)$ & $20.6(24.5)$ & $23.5(26.5)$ & $24.8(26.1)$ & $26.5(29.4)$ \\
\hline
\end{tabular}




\begin{tabular}{|c|c|c|c|c|c|}
\hline \multirow{2}{*}{ Characteristic } & \multicolumn{5}{|c|}{ Optimism } \\
\hline & Overall $(\mathrm{N}=33,326)$ & Quartile $1_{(\mathrm{n}=8,206)}$ & Quartile $2_{(\mathrm{n}=9,242)}^{e}$ & Quartile $\boldsymbol{3}^{f}(\mathrm{n}=6,103)$ & Quartile $4^{g}(\mathrm{n}=9,775)$ \\
\hline Mean diet AHEI (SD) & $51.3(13.1)$ & $49.5(12.9)$ & $50.9(13.3)$ & $52.3(12.9)$ & $52.6(13.0)$ \\
\hline Mean BMI (SD) & $26.0(5.6)$ & $26.1(5.9)$ & $26.0(5.6)$ & $25.8(5.4)$ & $25.9(5.5)$ \\
\hline \multicolumn{6}{|l|}{ Health conditions } \\
\hline Mean CESD-R (SD) & $5.0(3.8)$ & $7.8(4.4)$ & $5.2(3.2)$ & $4.3(2.9)$ & $3.0(2.7)$ \\
\hline $\begin{array}{l}\quad \text { Depression status } \\
\text { (CESD- } \mathrm{R} \geq 10 \text { or } \\
\text { physician diagnosed or } \\
\text { antidepressant use prior to } \\
\text { or in } 2004 \text { ), } \%\end{array}$ & 17.8 & 42.3 & 22.6 & 18.9 & 13.4 \\
\hline
\end{tabular}

${ }^{a}$ Values are either percentages or means and their SD.

${ }^{b}$ Values of categorical variables may not sum to $100 \%$ due to rounding or because of missing data.

${ }^{c}$ Optimism was measured using the Life Orientation Test - Revised (LOT-R).

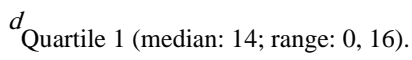

e Quartile 2 (median: 19; range: 17, 20).

$f$ Quartile 3 (median: 22; range: 21, 22).

$g_{\text {Quartile }} 4$ (median: 24; range: 23, 24).

MET/week, Metabolic equivalent of task hours per week; AHEI, Alternate Healthy Eating Index; CESD-R, Center for Epidemiologic Studies Depression Scale Revised. 
Table 2.

Rate Ratios and 95\% CI for the Association Between Optimism and Healthy Aging (2004 to 2012; N=33,326)

\begin{tabular}{|c|c|c|c|c|c|c|}
\hline Models & Continuous optimism score $^{a}$ & $\begin{array}{c}\text { Quartile 1 } \\
(\mathrm{n}=8,206) \\
(\mathrm{n}=1,206 \\
\text { cases })\end{array}$ & $\begin{array}{c}\text { Optimism } \\
\text { Quartile 2 } \\
\text { (n=9,242) } \\
\text { (n=1,687 cases) }\end{array}$ & $\begin{array}{c}\text { Quartile } 3 \\
(n=6,103) \\
(n=1,354 \text { cases })\end{array}$ & $\begin{array}{c}\text { Quartile } 4 \\
(n=9,775) \\
(n=2,576 \text { cases })\end{array}$ & $P$-trend \\
\hline Age-adjusted model 1 & $1.22(1.19,1.25)$ & $\operatorname{ref}(1.0)$ & $1.26(1.18,1.34)$ & $1.47(1.37,1.57)$ & $1.69(1.59,1.79)$ & $<0.001$ \\
\hline Model $2^{b}$ & $1.08(1.06,1.10)$ & $\operatorname{ref}(1.0)$ & $1.07(1.00,1.14)$ & $1.16(1.09,1.24)$ & $1.23(1.16,1.30)$ & $<0.001$ \\
\hline Model $3^{c}$ & $1.07(1.05,1.10)$ & $\operatorname{ref}(1.0)$ & $1.06(1.00,1.13)$ & $1.14(1.07,1.21)$ & $1.21(1.14,1.28)$ & $<0.001$ \\
\hline
\end{tabular}

Notes: Boldface indicates statistical significance $(p<0.05)$.

${ }^{a}$ Per 1 SD increase in Life Orientation Test - Revised (LOT-R) score.

$b_{\text {Model }} 2$ adds potential confounding factors to Model 1: physical function at baseline, age, race, marital status, nurses' education, husbands' education, census tract median income, and current/past depression (doctor-diagnosed depression, anti-depressant medication use, or high depressive symptoms).

${ }^{c}$ Model 3 adds possible intermediates (which could also be confounders) to Model 2: BMI, smoking status, physical activity, alcohol consumption, physical examination for screening purposes, diet (Alternate Healthy Eating Index [AHEI]). 
Table 3.

Rate Ratios and 95\% CIs for the Association Between Optimism and Healthy Aging by Race (2004 to 2012; $\mathrm{N}=32,981)$

\begin{tabular}{|c|c|c|c|c|c|c|}
\hline \multicolumn{7}{|c|}{ Optimism } \\
\hline Variable & Continuous optimism score ${ }^{a}$ & Quartile 1 & Quartile 2 & Quartile 3 & Quartile 4 & $p$-trend \\
\hline \multicolumn{7}{|l|}{ Black women $(n=354)$} \\
\hline $\mathrm{n}$ (cases) & & $54(5)$ & $103(8)$ & $67(13)$ & $130(26)$ & \\
\hline Age-adjusted model 1 & $1.48(1.04,2.09)$ & $\operatorname{ref}(1.0)$ & $0.86(0.31,2.38)$ & $2.03(0.81,5.07)$ & $2.00(0.84,4.77)$ & $\mathbf{0 . 0 3}$ \\
\hline Model $2^{b}$ & $1.42(1.01,1.98)$ & $\operatorname{ref}(1.0)$ & $1.00(0.39,2.55)$ & $1.95(0.84,4.48)$ & $1.93(0.85,4.41)$ & 0.04 \\
\hline Model $3^{c}$ & $1.36(0.97,1.91)$ & $\operatorname{ref}(1.0)$ & $1.07(0.42,2.71)$ & $1.95(0.86,4.42)$ & $1.82(0.78,4.24)$ & 0.08 \\
\hline \multicolumn{7}{|l|}{ White Women $(n=32,627)$} \\
\hline $\mathrm{n}$ (cases) & & $8,059(1,191)$ & $9,014(1,656)$ & $5,978)(1,332)$ & $9,576(2,525)$ & \\
\hline Age-adjusted model 1 & $1.22(1.19,1.25)$ & $\operatorname{ref}(1.0)$ & $1.26(1.18,1.34)$ & $1.47(1.37,1.57)$ & $1.69(1.59,1.79)$ & $<0.001$ \\
\hline Model $2^{b}$ & $1.08(1.06,1.10)$ & $\operatorname{ref}(1.0)$ & $1.07(1.00,1.13)$ & $1.17(1.09,1.24)$ & $1.23(1.16,1.30)$ & $<0.001$ \\
\hline Model $3^{c}$ & $1.07(1.05,1.10)$ & $\operatorname{ref}(1.0)$ & $1.05(0.99,1.12)$ & $1.14(1.07,1.22)$ & $1.20(1.14,1.28)$ & $<0.001$ \\
\hline
\end{tabular}

Notes: Boldface indicates statistical significance $(p<0.05)$. Due to power constraints, all continuous covariates were included in race-stratified models, but categorical covariates were dichotomized as follows: marital status (married/other); nurses' education (registered nurse/other); and husbands' education (college graduate or graduate school/other); census-tract median income (above median [\$61,429]/below median); physical activity ( $\geq 18$ metabolic equivalent of task hours per week/other); alcohol intake (none/ >0-15+ g/d); smoking (ever/never).

${ }^{a}$ Per 1 SD increase in Life Orientation Test - Revised (LOT-R) score.

$b_{\text {Model }} 2$ adds potential confounders to Model 1: physical functioning at baseline, age, race, marital status, nurses' education, husbands' education, census tract median income, and current/past depression (doctor-diagnosed depression, anti-depressant medication use, or high depressive symptoms).

${ }^{c}$ Model 3 adds to Model 2 potential intermediates (which may also be confounders): BMI, smoking status, physical activity, alcohol consumption, physical examination for screening purposes, diet (Alternate Healthy Eating Index). 